\title{
VALIDATION OF COMBINATORIAL EVALUATION OF STRAIN-GAUGE AMPLIFIER LINEARITY
}

\author{
M. Hiti ${ }^{1}$ \\ ${ }^{1}$ Slovenian National Building and Civil Engineering Institute (ZAG), Ljubljana, Slovenia, miha.hiti@zag.si
}

\begin{abstract}
:
This paper describes a validation of a combinatorial calibration technique based calibration procedure for strain-gauge amplifier calibration. The deviation between two strain-gauge amplifiers at calibration using the combinatorial technique is compared to the deviation between the same two amplifiers at calibration on a force calibration machine as a transducer-amplifier chain under unchanged conditions, serving as an amplifier linearity comparator. This enables the validation to be confirmed with a suitable expanded measurement uncertainty of $3 \mathrm{nV} / \mathrm{V}$.
\end{abstract}

Keywords: strain-gauge amplifier; calibration; combinatorial technique; validation

\section{INTRODUCTION}

The calibration of strain-gauge amplifiers (bridge amplifiers) used in measuring chains for force, torque and pressure measurements is potentially subjected to large measurement uncertainties when calibrated with traditional voltage ratio $(\mathrm{mV} / \mathrm{V})$ standards - bridge standards. In previous publication [1] we presented an alternative calibration procedure which reduces the calibration uncertainty to a more acceptable level by calibrating the bridge amplifier at a single reference point with a traditional bridge standard and additionally performing linearity evaluation between the relative $0 \mathrm{mV} / \mathrm{V}$ and the calibrated

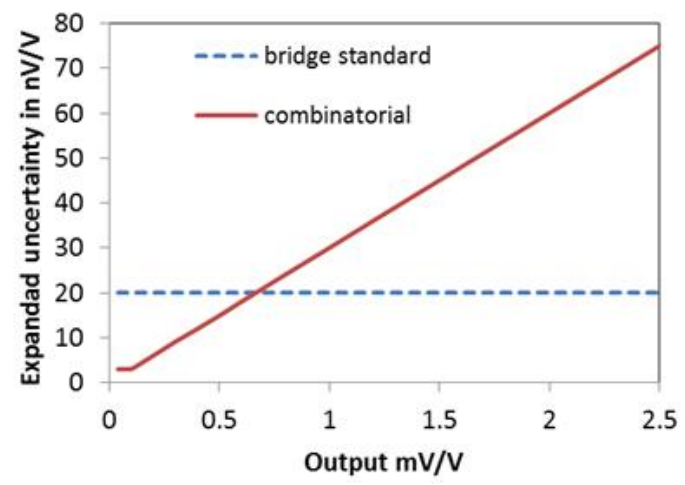

reference point using the ZAG LinCheck combinatorial system [2]. This procedure has already been offered to customers as a calibration service within ISO/IEC 17025 accredited scope with an expanded relative measurement uncertainty of $3 \mathrm{nV} / \mathrm{V}$ or $0.003 \%$ (whichever is higher) in the range from $0.05 \mathrm{mV} / \mathrm{V}$ to $2.5 \mathrm{mV} / \mathrm{V}$ for positive and negative voltage ratios, covering the typically required range, Figure 1.

One of the requirements at assessment of a calibration laboratory's competence regarding accredited services is the validation of calibration procedures. While the combinatorial procedure has been reproduced also by other laboratories to verify the linearity of amplifiers with comparable results [3], the validation of the procedure by comparison with a typical best-available calibrated voltage ratio standards is not sufficient. The measurement uncertainty of the latter exceeds the potentially achievable measurement uncertainty of the combinatorial calibration procedure even after the best available calibration procedure for bridge standard calibration has been improved, reducing the expanded uncertainty for bridge standard calibration from $10 \mathrm{nV} / \mathrm{V}$ to $5 \mathrm{nV} / \mathrm{V}$ (for $k=2$ ) [4]. Lower uncertainties for linearity evaluation were not possible until recently, when a cascaded inductive voltage divider setup was presented for bridge amplifier linearity evaluation, achieving expanded uncertainty of $2 \mathrm{nV} / \mathrm{V}$ [5] and offering $10^{7}$ steps within the $\pm 5 \mathrm{mV} / \mathrm{V}$ range.

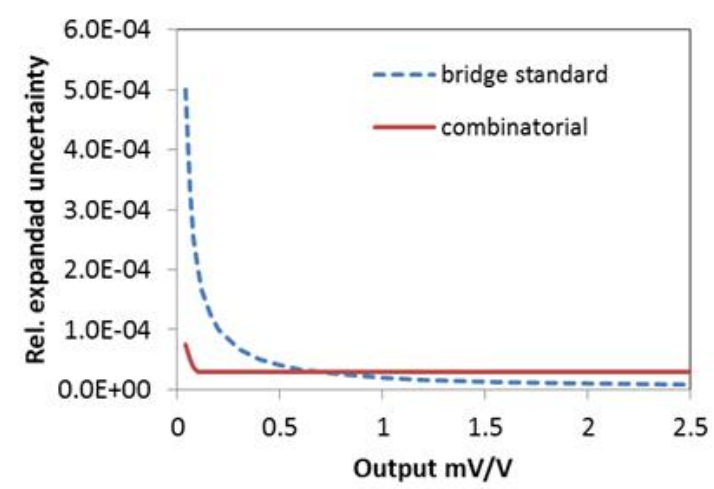

Figure 1: Expanded measurement uncertainty for bridge amplifier calibration with traditional bridge standards calibration and combinatorial procedure (left) and expressed as relative expanded uncertainty (right) 
A direct comparison of the combinatorial procedure with the newly presented unique cascaded inductive voltage divider setup could provide the reference needed for the validation, but in this paper we present a different approach: to use a force calibration machine as a relative standard for voltage ratio linearity comparison. Force calibration machines are widely available at NMIs and calibration laboratories and offer expanded measurement uncertainties of generated force down to $0.001 \%$. When calibration of a single force transducer is repeated with different bridge amplifiers under unchanged conditions, the amplifier linearity could be compared by analysing the calibration results, and thus acting as a comparator for relative bridge linearity evaluation.

\section{DESCRIPTION OF THE WORK}

Previous investigation of calibration of the same transducer with different amplifiers has shown that the agreement between comparable amplifiers of the same type and supply voltage can be within $0.003 \%$ [6], Figure 2.

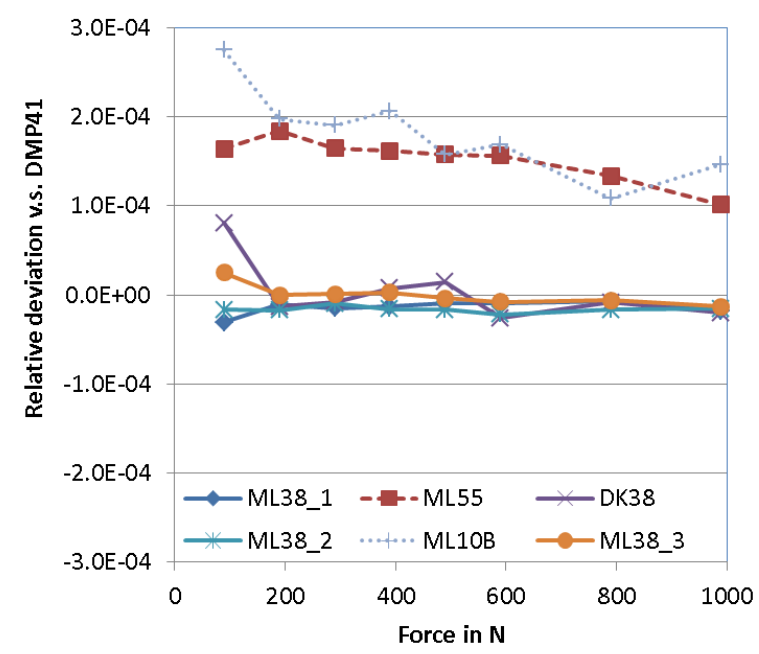

Figure 2: Results of deviation of transducer calibration with different amplifiers compared to an HBM DMP41 (amplifier sensitivity errors corrected)

In these investigations, the same force transducer $(1 \mathrm{kN})$ was calibrated with different amplifiers: $225 \mathrm{~Hz}$ carrier frequency type amplifiers HBM DMP41, HBM ML38B and HBM DK38, $4.8 \mathrm{k} \mathrm{Hz}$ carrier frequency type amplifier HBM ML55B and a DC excitation type amplifier HBM ML10B. Each of these amplifiers was sequentially connected to the same transducer and measurements were performed in the same $1 \mathrm{kN}$ dead-weight force calibration machine (FCM) with $<0.002 \%$ reproducibility (the accredited CMC of the force calibration machine is $0.005 \%$, but this value includes many uncertainty contributions which are irrelevant in the case of comparative measurements). The transducer and the force calibration machine remained in unchanged configuration, as the aim of this investigation was to compare the effect of different amplifiers on the calibration result.

Measurements with one ML38B amplifier were done at the beginning of the investigation, at the end of the investigation, and between measurements with other amplifiers to verify the stability and reproducibility of the measurement setup and measurement procedure itself. Before each measurement series, each amplifier was calibrated by a calibrated bridge standard HBM K3608 with expanded measurement uncertainty of $20 \mathrm{nV} / \mathrm{V}$ for the $225 \mathrm{~Hz}$ carrier frequency, $330 \mathrm{nV} / \mathrm{V}$ for $4.8 \mathrm{kHz}$ carrier frequency, and $200 \mathrm{nV} / \mathrm{V}$ for DC excitation voltage.

The deviation of resulting characteristics in Figure 2 shows the indication deviation of each amplifier from a reference characteristic - in this case the characteristic of DMP41 amplifier. But these measurements offered also the possibility to compare the linearity of the amplifiers, as the relevant information can be extracted from the results. Results suggested that the amplifier linearity could be very good, as can typically also be observed by calibration with voltage ratio standards, albeit with a high measurement uncertainty $(>20 \mathrm{nV} / \mathrm{V})$, and the much lower relative uncertainty of the force measurement setup could help to prove these assumption.

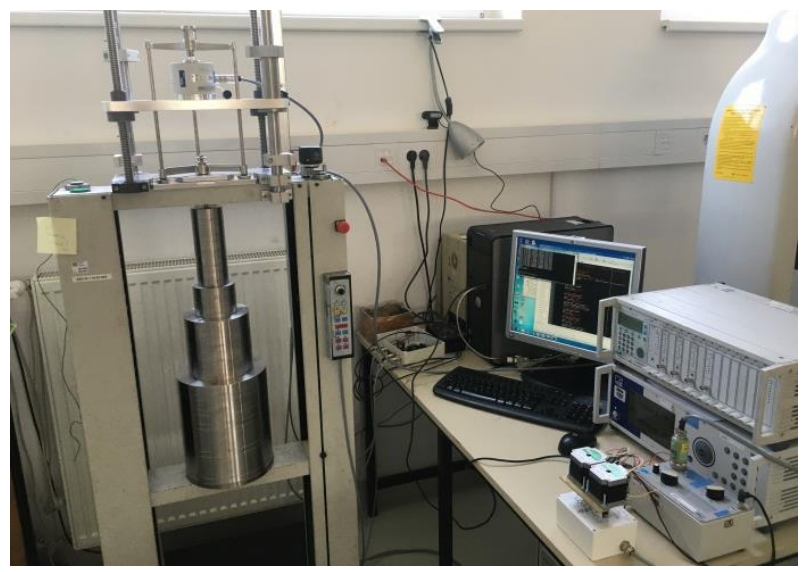

Figure 3: Measurement setup with $1 \mathrm{kN}$ force transducer positioned in $1 \mathrm{kN}$ force calibration machine with HBM DMP41 amplifier and HBM MGCplus system with ML38B amplifier. A HBM K3608 bridge standard and a ZAG LinCheck system for combinatorial calibration are also shown.

Based on these results the measurements with the $1 \mathrm{kN}$ HBM Z30A-TOP transducer in the $1 \mathrm{kN}$ dead-weight force calibration machine were repeated for the HBM DMP41 and HBM ML38B amplifiers under more stringent conditions with the aim to investigate the comparability of linearity of these amplifiers, Figure 3. 


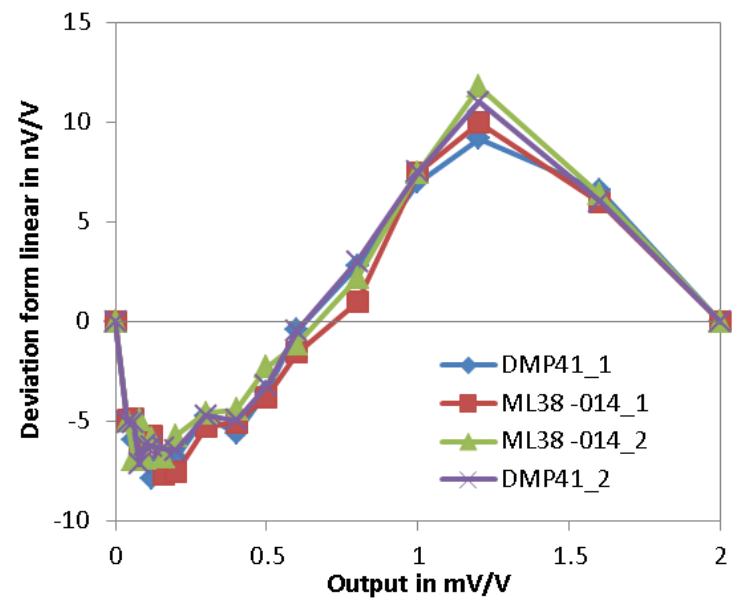

Figure 4: Deviation of two different amplifiers from linear characteristics $(0 \mathrm{mV} / \mathrm{V}-2 \mathrm{mV} / \mathrm{V})$ for the same force transducer in the same FCM - results normalized to $2 \mathrm{mV} / \mathrm{V}$

For the best possible reproducibility of the force steps, the force transducer was positioned in a single rotational position, its position was not changed during the whole process, and the loading frame of the force calibration machine (the first $10 \mathrm{~N}$ weight) was never unloaded to keep the force introduction as repeatable as possible. This way most uncertainty sources arising from the force calibration machine and the force transducer have been significantly reduced or eliminated, such as the effects of machine and transducer alignment, reproducibility of force transducer in different transducer rotations and the effect of change in load application. By eliminating most uncertainty sources, the uncertainty of force results mainly from the repeatability of the relative force steps. The contributions of mass and gravitational field only influence the process as relative terms during the short measurement period and their exact absolute values do not even have to be known. To reduce the effect of force transducer creep, the force calibration machine was controlled in automatic mode with constant time intervals between each measurement and the same pre-defined loading profile for all measurements. Both amplifiers were constantly powered on and a dummy load was used to simulate the transducer load when the actual $1 \mathrm{kN}$ force transducer was occupied by the other amplifier, to keep the operating conditions of the amplifiers as constant as possible. Amplifier parameters were set to the same values on both amplifiers: $5 \mathrm{~V}$ excitation voltage, $0.1 \mathrm{~Hz}$ Bessel filter settings. The selected time interval between measurements was $60 \mathrm{~s}$. The final measurements were done sequentially within two hours. Measurements were performed in the range from $10 \mathrm{~N}$ to $990 \mathrm{~N}$ in an AB-B-A sequence (DMP41-ML38B-ML38BDMP41). The same amplifiers were later calibrated also with the ZAG LinCheck system for combinatorial calibration.

\section{RESULTS}

The results of calibration of the $1 \mathrm{kN}$ transducer with the DMP41 and the ML38B are shown in Figure 4 , where the characteristic sensitivities are normalized to $2 \mathrm{mV} / \mathrm{V}$. The data obtained from the calibration of the amplifiers in the FCM includes also the transducer nonlinearity contribution, which is in this case unknown, so the nonlinearity of the amplifier cannot be simply extracted from the results. However, the difference in nonlinearity results can be used as a reference for the comparison as it should depend mainly on the amplifier, if other system parameters are kept constant.

It can be seen in Figure 4 that both measured DMP41 series and both ML38B series are in good agreement, with the largest deviation between all series being $3 \mathrm{nV} / \mathrm{V}$ (at $1.2 \mathrm{mV} / \mathrm{V}$ output ratio). The deviation between average ML38B and average DMP41 characteristics is shown in Figure 5 as absolute and relative deviation.

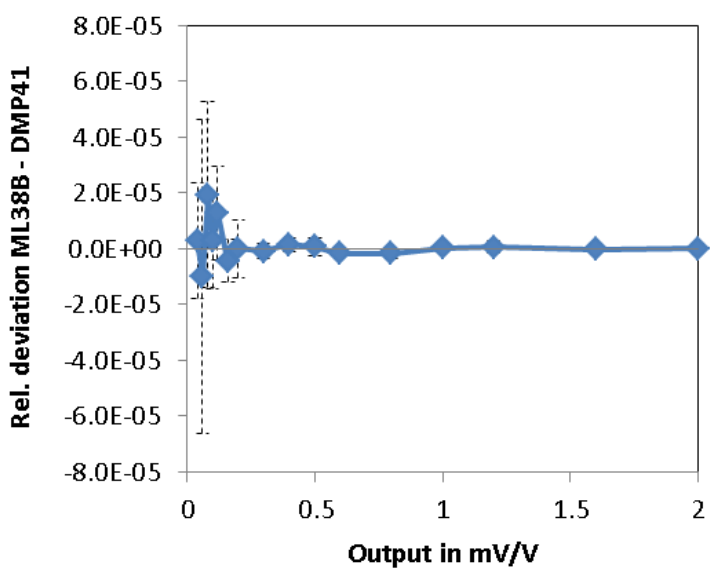

Figure 5: Deviation between average ML38B and average DMP41 as absolute deviation (left) and relative deviation (right). Standard uncertainty of the deviation is also shown with error bars (contributions of repeatability and resolution). 


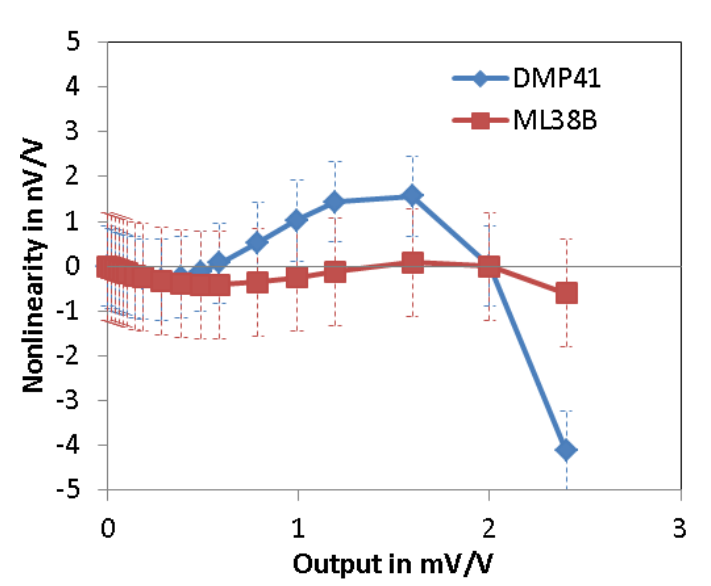

Figure 6: Linearity evaluation of deviation of two different amplifiers from linear characteristics $(0 \mathrm{mV} / \mathrm{V}$ to $2 \mathrm{mV} / \mathrm{V}$ ) with the ZAG LinCheck system. Results adjusted at $2 \mathrm{mV} / \mathrm{V}$.

The result suggests that the linearity of the amplifiers agrees for the most part, with deviations of less than $2 \times 10^{-5}$. For the uncertainty attributed to the measured deviation, contributions of the repeatability of two series and contributions of amplifier resolution were taken into account. It is within $2 \mathrm{nV} / \mathrm{V}$ or up to $5 \times 10^{-5}$ for the lowest ratios.

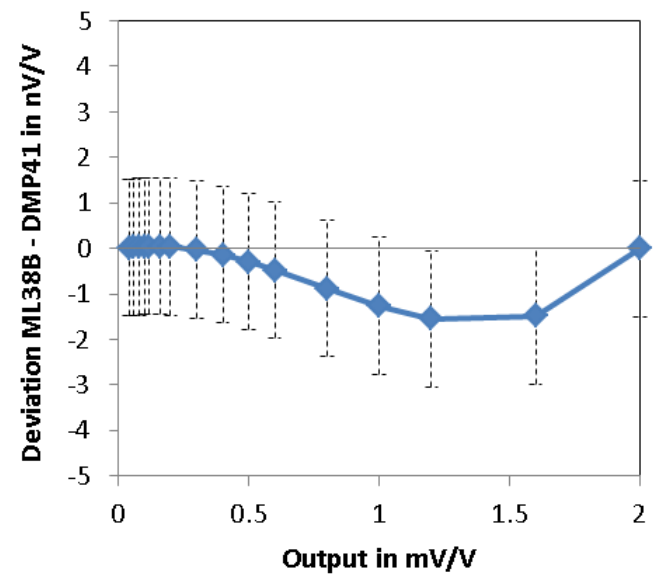

Immediately after the linearity measurements on the force calibration machine, both amplifiers were also evaluated with the ZAG LinCheck system employing the combinatorial method. The amplifier parameters were the same as during measurements on the force calibration machine, but the evaluated range was in this case up to $2.5 \mathrm{mV} / \mathrm{V}$, Fehler! Verweisquelle konnte nicht gefunden werden.. The resulting characteristics are shown with standard uncertainty of nonlinearity determination of $0.9 \mathrm{nV} / \mathrm{V}$ for DMP41 and $1.2 \mathrm{nV} / \mathrm{V}$ for ML38B. The nonlinearity results of both amplifiers have been adjusted to a $2 \mathrm{mV} / \mathrm{V}$ reference point.

For comparison with linearity results obtained by evaluation of the amplifiers on the force calibration machine, the difference between ML38B and DMP41 results was calculated, shown in Figure 7 as absolute and relative difference. The determined nonlinearity difference of the two amplifiers using combinatorial method up to $2 \mathrm{mV} / \mathrm{V}$ is within $2 \mathrm{nV} / \mathrm{V}$, below $2 \times 10^{-6}$ if expressed as relative deviation, with standard uncertainty up to $7.5 \times 10^{-5}$.

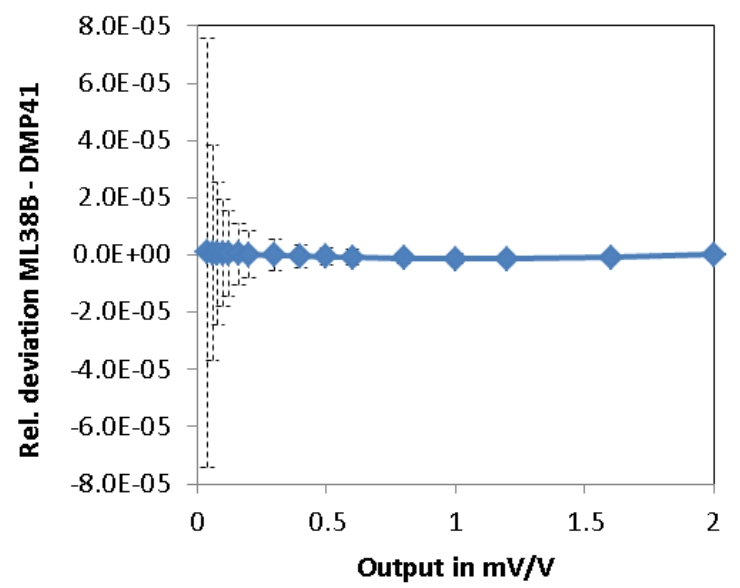

Figure 7: Deviation between ML38B and DMP41 as absolute deviation (left) and relative deviation (right) measured by employing combinatorial method (ZAG LinCheck). Standard uncertainty of the deviation is also shown with error bars.
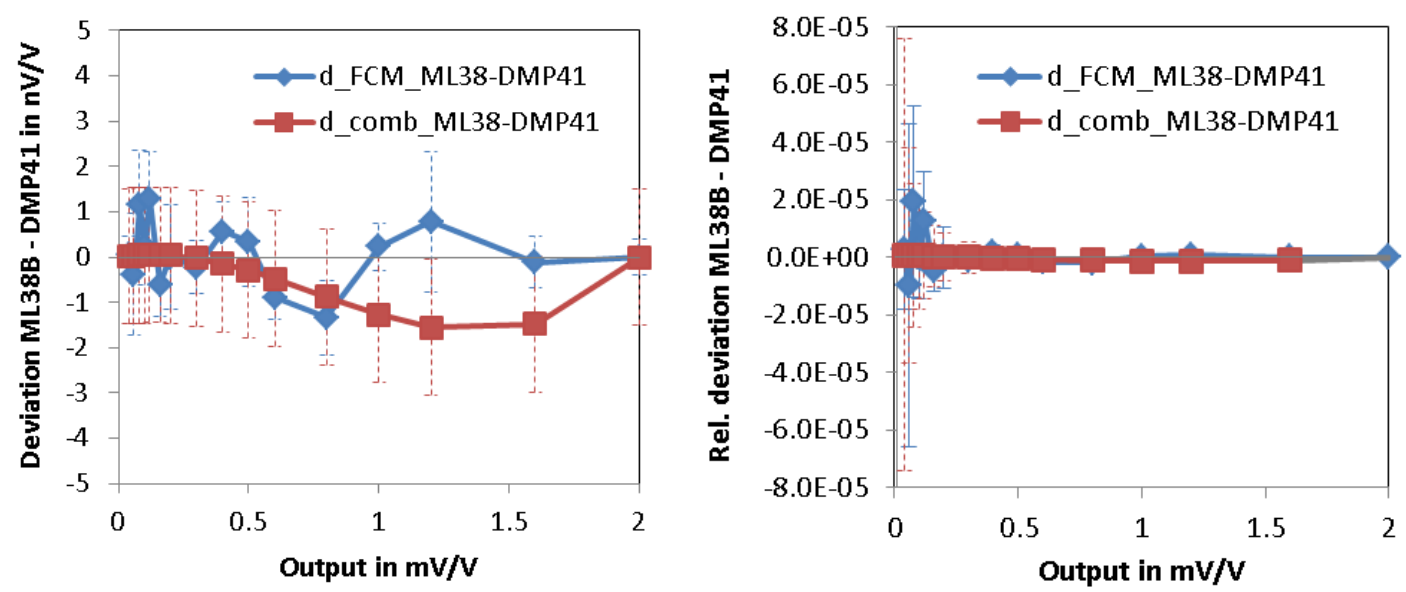

Figure 8: Comparison of nonlinearity deviation between ML38B and DMP41 determined in a FCM (FCM) and by combinatorial method (comb). Standard uncertainty of the deviation is shown with error bars. 
The comparison of both methods of linearity evaluation is shown in Figure 8. The figure shows the deviation in nonlinearity difference of both amplifiers, once obtained from calibration in the FCM and once obtained by combinatorial calibration. By comparing the results from both methods, it can be seen that the two methods agree to within their respective standard uncertainties.

\section{DISCUSSION}

The evaluation of the linearity of amplifiers with different methods produced comparable results. As the combinatorial method has already been demonstrated to produce comparable results to traditional linearity evaluation with bridge standards, with large uncertainty of the latter, the additional linearity comparison via force calibration machine confirms the suitability of the combinatorial calibration procedure for amplifier calibration. The expanded measurement uncertainty of the comparison was $3 \mathrm{nV} / \mathrm{V}$. This confirmation suggests, that it is possible to realize the linearity evaluation of $225 \mathrm{~Hz}$ carrier frequency amplifiers with the ZAG LinCheck system to within $2 \mathrm{nV} / \mathrm{V}$ expanded measurement uncertainty.

At the same time it also confirms the comparability of linearity determination by transducer calibration in a FCM if an amplifier with known linearity is available. In combination with force calibration machines offering low expanded uncertainties of generated force, the aim is to further improve the amplifier calibration to be able to subtract the amplifier from the calibration result of the force transducer under calibration, specifying only the transducer characteristics.

\section{SUMMARY}

This paper presents an example of a procedure to validate the combinatorial evaluation procedure for strain-gauge amplifier linearity evaluation. By using a single force calibration machine and a single force transducer with various bridge amplifiers, the force calibration system acts as a comparator to compare the linearity of amplifiers. The lower uncertainty of generated force enables better measurement uncertainties than offered by traditional bridge calibrators and is in line with the requirements for the combinatorial system $(3 \mathrm{nV} / \mathrm{V})$. The proposed procedure is suitable for the confirmation of performance of the combinatorial calibration procedure and should enable the validation and application of the procedure in ISO/IEC 17025 accredited calibration laboratories.

\section{REFERENCES}

[1] M. Hiti, "Reducing the uncertainty of strain gauge amplifier calibration", ACTA IMEKO, vol. 6, no. 4, pp. 69-74, 2017. Online [accessed 20200821]: https://acta.imeko.org/index.php/actaimeko/article/view/IMEKO-ACTA06\%20\%282017\%29-04-11/pdf

[2] M. Hiti, "Resistor network for linearity check of voltage ratio meters by combinatorial technique", Meas. Sci Technol., vol. 26, no. 5, 2015.

[3] M. M. Schäck: "Long Term Proven and Optimized High-Precision $225 \mathrm{~Hz}$ Carrier Frequency Technology in a Modern and Universal Data Acquisition System", Proc. IMEKO 23rd TC3, 13th TC5 and 4th TC22 International Conference, Helsinki, Finland, 30 May to 1 June 2017.

[4] M. F. Beug, A. Kölling, H. Moser, "A new calibration transformer and measurement setup for bridge standard calibrations up to $5 \mathrm{kHz}$ ", IEEE Transactions on Instrumentation and Measurement, vol. 66, no. 6, pp. 1531-1538, June 2017.

[5] M. F. Beug, H. Moser, A. Kölling: "Bridge Amplifier Linearity Investigation with a Cascaded Inductive Voltage Divider Setup", J. Phys.: Conf. Ser. 1065 042010, 2018.

[6] M. Hiti: "Analysis of exchanging of measuring amplifiers on force transducer calibration results", ERK 2017, Portorož, Slovenia, 25 - 26 September 2017. 